Article

\title{
New Sesquiterpenoids and Anti-Platelet Aggregation Constituents from the Rhizomes of Curcuma zedoaria
}

\author{
Jih-Jung Chen ${ }^{1,2, *,+}$, Tung-Han Tsai ${ }^{1}$, Hsiang-Ruei Liao ${ }^{3,+}{ }^{,}$Li-Chai Chen ${ }^{1,4,+}$, \\ Yueh-Hsiung Kuo ${ }^{5,6,+}$, Ping-Jyun Sung ${ }^{7}$, Chun-Lin Chen ${ }^{8}$ and Chun-Sheng Wei ${ }^{4,+}$ \\ 1 Department of Pharmacy, Tajen University, Pingtung 907, Taiwan; jjc5711@yahoo.com.tw (T.-H.T.); \\ icupdrab@gmail.com (L.-C.C.) \\ 2 Department of Medical Research, China Medical University Hospital, China Medical University, \\ Taichung 404, Taiwan \\ 3 Graduate Institute of Natural Products, College of Medicine, Chang Gung University, Taoyuan 333, Taiwan; \\ liaoch@mail.cgu.edu.tw \\ 4 Department of Pharmacy, Zuoying Branch of Kaohsiung Armed Forces General Hospital, \\ Kaohsiung 813, Taiwan; pharmacist@ngh.com.tw \\ 5 Department of Chinese Pharmaceutical Sciences and Chinese Medicine Resources, China Medical University, \\ Taichung 404, Taiwan; kuoyh@mail.cmu.edu.tw \\ 6 Department of Biotechnology, Asia University, Taichung 413, Taiwan \\ 7 National Museum of Marine Biology and Aquarium, Pingtung 944, Taiwan; pjsung@nmmba.gov.tw \\ 8 Department of Biological Sciences, National Sun Yat-sen University, Kaohsiung 804, Taiwan; \\ chunlinchen@mail.nsysu.edu.tw \\ * Correspondence: jjchen@tajen.edu.tw; Tel.: +886-8-7624-002 (ext. 2827); Fax: +886-8-7624-002 (ext. 5121) \\ $\dagger$ These authors contributed equally to this work.
}

Academic Editor: Derek J. McPhee

Received: 16 September 2016; Accepted: 12 October 2016; Published: 17 October 2016

\begin{abstract}
Two new sesquiterpenoids-13-hydroxycurzerenone (1) and 1-oxocurzerenone (2)-have been isolated from the rhizomes of Curcuma zedoaria, together with 13 known compounds (3-15). The structures of two new compounds were determined through spectroscopic and MS analyses. Among the isolated compounds, 13-hydroxycurzerenone (1), 1-oxocurzerenone (2), curzerenone (3), germacrone (4), curcolone (5), procurcumenol (6), ermanin (7), curcumin (8), and a mixture of stigmast-4-en-3,6-dione (12) and stigmasta-4,22-dien-3,6-dione (13) exhibited inhibition (with inhibition $\%$ in the range of $21.28 \%-67.58 \%$ ) against collagen-induced platelet aggregation at $100 \mu \mathrm{M}$. Compounds 1, 5, 7, 8, and the mixture of 12 and 13 inhibited arachidonic acid (AA)-induced platelet aggregation at $100 \mu \mathrm{M}$ with inhibition $\%$ in the range of $23.44 \%-95.36 \%$.
\end{abstract}

Keywords: Curcuma zedoaria; Zingiberaceae; rhizomes; sesquiterpenoids; anti-platelet aggregation

\section{Introduction}

Curcuma zedoaria (Christm.) Rosc. (Zingiberaceae) is a perennial rhizomatous herb indigenous to Bangladesh, Sri Lanka, and India, and is also widely cultivated in China, Japan, Brazil, Nepal, and Thailand. Various sesquiterpenoids [1-4], curcuminoids [3], benzenoids [3], and their derivatives were isolated from this plant in previous studies. Many of these compounds exhibit anti-inflammatory [1], anti-babesial [2], cytotoxic [3], and anti-fungal [4] activities.

In our studies of medicinal plants for in vitro anti-platelet aggregation activity, C. zedoaria was found to be an active species. The $\mathrm{MeOH}$ extract of the rhizomes of $C$. zedoaria displayed antiplatelet aggregation activity. Figure 1 illustrates the structures of two new sesquiterpenoids, 13-hydroxycurzerenone (1) and 1-oxocurzerenone (2). Thirteen known compounds (3-15) have been isolated and identified from the rhizomes of $C$. zedoaria, and their structures are depicted in Figure 2. 
This paper describes the structural elucidation of new compounds $\mathbf{1}$ and $\mathbf{2}$ and the anti-platelet aggregation activities of the isolates.



1

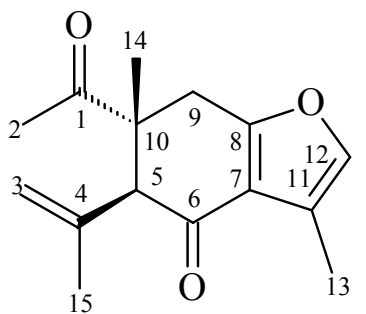

2

Figure 1. The chemical structures of new compounds $\mathbf{1}$ and $\mathbf{2}$ isolated from C. zedoaria.<smiles>C=C[C@]1(C)Cc2occ(C)c2C(=O)[C@H]1C(=C)C</smiles>

3<smiles>CC1=CC(=O)C(=C(C)C)C[C@H]2[C@@H](C)CC[C@@H]12</smiles><smiles>CC1=CCC(=C(C)C)C(=O)CC(C)=CCC1</smiles>

4<smiles>CC1=C2C(=O)c3c(C)coc3C[C@]2(C)[C@H](O)CC1</smiles>

5<smiles>COc1cc(/C=C/C(=O)CC(=O)/C=C/c2ccc(O)c(OC)c2)ccc1O</smiles><smiles>CCCCCCCCCCCCCCCCCCCCCCCCCCCCCCC(=O)OCC(COC(=O)CCCCCCCC)OC(=O)CCCCCCCCCCCC</smiles>

Figure 2. Cont. 

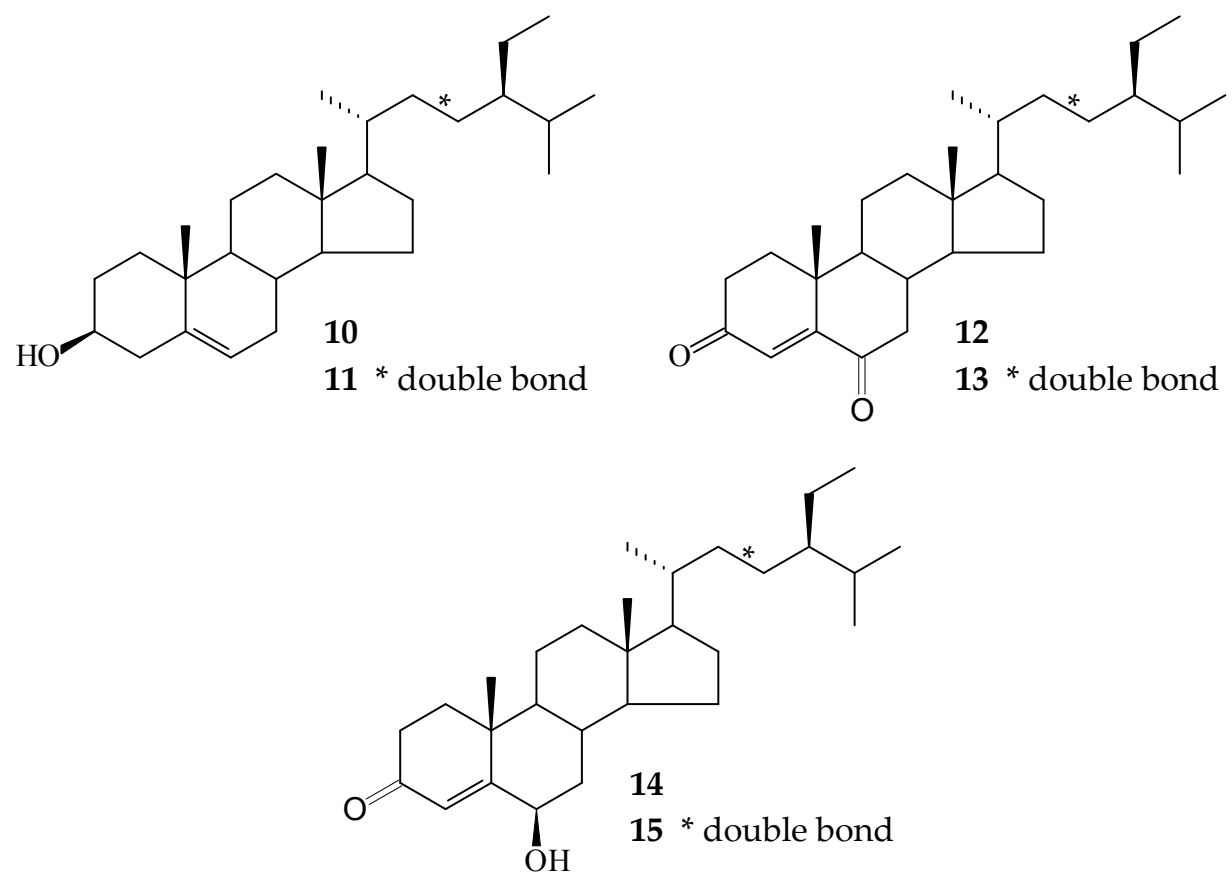

Figure 2. The chemical structures of known compounds 3-15 isolated from C. zedoaria.

\section{Results and Discussion}

13-Hydroxycurzerenone (1) was isolated as a yellowish oil. Its molecular formula $\left(\mathrm{C}_{15} \mathrm{H}_{18} \mathrm{O}_{3}\right)$ was determined on the basis of the quasi-molecular ion at $m / z 269.1157\left([\mathrm{M}+\mathrm{Na}]^{+}\right.$, calcd. for $\mathrm{C}_{15} \mathrm{H}_{18} \mathrm{O}_{3} \mathrm{Na}$ : 269.1154) in the high-resolution electrospray ionization mass spectrometry (HR-ESI-MS) spectrum (positive-ion mode) and was supported by the ${ }^{1} \mathrm{H}-,{ }^{13} \mathrm{C}-$, and DEPT NMR data. The presence of a conjugated carbonyl group was revealed by the band at $1678 \mathrm{~cm}^{-1}$ in the IR spectrum, which was confirmed by the resonance at $\delta_{C} 194.0$ in the ${ }^{13} \mathrm{C}$-NMR spectrum. The IR of $\mathbf{1}$ also showed OH absorption at $3450 \mathrm{~cm}^{-1}$. The ${ }^{1} \mathrm{H}-\mathrm{NMR}$ spectrum of $\mathbf{1}$ showed resonances for an aromatic proton $\left(\delta_{\mathrm{H}} 7.09(1 \mathrm{H}, \mathrm{s}, \mathrm{H}-12)\right)$, a hydroxymethyl group $\left(\delta_{\mathrm{H}} 4.86(2 \mathrm{H}, \mathrm{s}, \mathrm{H}-13)\right)$, a methyl group $\left(\delta_{\mathrm{H}} 1.19(3 \mathrm{H}\right.$, $\mathrm{s}, \mathrm{H}-14))$, a vinyl group $\left(\delta_{\mathrm{H}} 4.96(1 \mathrm{H}, \mathrm{d}, J=17.5 \mathrm{~Hz}, \mathrm{H}-2), 4.97(1 \mathrm{H}, \mathrm{d}, J=11.0 \mathrm{~Hz}, \mathrm{H}-2), 5.82(1 \mathrm{H}\right.$, $\mathrm{dd}, J=17.5,11.0 \mathrm{~Hz}, \mathrm{H}-1))$, a prop-1-en-2-yl group $\left(\delta_{\mathrm{H}} 1.84(3 \mathrm{H}, \mathrm{s}, \mathrm{H}-15), 4.76(1 \mathrm{H}, \mathrm{s}, \mathrm{H}-3), 5.01\right.$ $(1 \mathrm{H}, \mathrm{s}, \mathrm{H}-3))$, a methine proton $\left(\delta_{\mathrm{H}} 3.02(1 \mathrm{H}, \mathrm{s}, \mathrm{H}-5)\right)$, and two methylene protons $(\delta 2.79(1 \mathrm{H}, \mathrm{d}$, $J=17.5 \mathrm{~Hz}, \mathrm{H}-9), 2.91(1 \mathrm{H}, \mathrm{d}, J=17.5 \mathrm{~Hz}, \mathrm{H}-9))$. Comparison of the ${ }^{1} \mathrm{H}-$ and ${ }^{13} \mathrm{C}-\mathrm{NMR}$ data (Table 1$)$ of 1 with those of curzerenone (3) $[5,6]$ suggested that their structures are closely related, except that the 11-hydroxymethyl group $\left[\delta_{\mathrm{H}} 4.86(2 \mathrm{H}, \mathrm{s}, \mathrm{H}-13) ; \delta_{\mathrm{C}} 52.2(\mathrm{C}-13)\right]$ of 1 replaced the 11-methyl group of curzerenone $(3)[5,6]$. This was supported by HMBC correlation between $\mathrm{H}-13\left(\delta_{\mathrm{H}} 4.86\right)$ and C-7 $\left(\delta_{\mathrm{C}}\right.$ $120.2), \mathrm{C}-11\left(\delta_{\mathrm{C}} 116.0\right)$, and $\mathrm{C}-12\left(\delta_{\mathrm{C}} 139.6\right)$, and NOESY correlations between $\mathrm{H}-13\left(\delta_{\mathrm{H}} 4.86\right)$ and $\mathrm{H}-12$ $\left(\delta_{\mathrm{H}} 7.09\right)$. The NOESY cross-peaks (Figure 3$)$ between $\mathrm{H}-1\left(\delta_{\mathrm{H}} 5.82\right) / \mathrm{H}-5\left(\delta_{\mathrm{H}} 3.02\right), \mathrm{H}-1 / \mathrm{H}_{\alpha}-9\left(\delta_{\mathrm{H}} 2.79\right)$, $\mathrm{H}-14\left(\delta_{\mathrm{H}} 1.19\right) / \mathrm{H}_{\mathrm{a}}-3\left(\delta_{\mathrm{H}} 5.01\right)$, and $\mathrm{H}-14 / \mathrm{H}_{\beta}-9\left(\delta_{\mathrm{H}} 2.91\right)$ suggested that the 10 -vinyl group, $\mathrm{H}-5$, and $\mathrm{H}_{\alpha}-9$ are $\alpha$-oriented, and the 10-methyl group, C-5 prop-1-en-2-yl group, and $\mathrm{H}_{\beta}-9$ are $\beta$-oriented. The full assignments of ${ }^{1} \mathrm{H}$ - and ${ }^{13} \mathrm{C}-\mathrm{NMR}$ resonances were supported by ${ }^{1} \mathrm{H}-{ }^{1} \mathrm{H}$ COSY, DEPT, HSQC, NOESY (Figure 3), and HMBC (Figure 3) spectral analyses. On the basis of the above data, the structure of $\mathbf{1}$ was elucidated as 13-hydroxy-curzerenone.

1-Oxocurzerenone (2) was isolated as a yellowish oil. The ESI-MS afforded the quasi-molecular ion $[\mathrm{M}+\mathrm{Na}]^{+}$at $m / z 269$, implying a molecular formula of $\mathrm{C}_{15} \mathrm{H}_{18} \mathrm{O}_{3} \mathrm{Na}$, which was confirmed by the HR-ESI-MS $\left(m / z 269.1153[\mathrm{M}+\mathrm{Na}]^{+}\right.$, calcd. 269.1154). The presence of two carbonyl groups was revealed by the bands at 1675 and $1706 \mathrm{~cm}^{-1}$ in the IR spectrum, and was confirmed by the resonances at $\delta_{\mathrm{C}} 194.0$ and 212.1 in the ${ }^{13} \mathrm{C}-\mathrm{NMR}$ spectrum. The ${ }^{1} \mathrm{H}$ - and ${ }^{13} \mathrm{C}-\mathrm{NMR}$ data (Table 1 ) of 2 were similar to those of curzerenone (3) [5,6], except that the 10-acetyl group $\left(\delta_{\mathrm{H}} 2.13(3 \mathrm{H}, \mathrm{s}, \mathrm{H}-2) ; \delta_{\mathrm{C}}\right.$ 
$25.6(\mathrm{C}-2), 212.1(\mathrm{C}-1))$ of 2 replaced the 10-vinyl group of curzerenone (3) [5,6]. This was supported by HMBC correlations between H-2 $\left(\delta_{\mathrm{H}} 2.13\right)$ and C-1 $\left(\delta_{\mathrm{C}} 212.1\right)$ and $\mathrm{C}-10\left(\delta_{\mathrm{C}} 46.2\right)$, and NOESY correlations between $\mathrm{H}-2\left(\delta_{\mathrm{H}} 2.13\right)$ and $\mathrm{H}-5\left(\delta_{\mathrm{H}} 3.22\right)$ and $\mathrm{H}_{\alpha}-9\left(\delta_{\mathrm{H}} 2.94\right)$. The NOESY cross-peaks (Figure 4) between $\mathrm{H}-2\left(\delta_{\mathrm{H}} 2.13\right) / \mathrm{H}-5\left(\delta_{\mathrm{H}} 3.22\right), \mathrm{H}-2 / \mathrm{H}_{\alpha}-9\left(\delta_{\mathrm{H}} 2.94\right), \mathrm{H}-14\left(\delta_{\mathrm{H}} 1.18\right) / \mathrm{H}-3\left(\delta_{\mathrm{H}} 5.01\right)$, and $\mathrm{H}-14 / \mathrm{H}_{\beta}-9\left(\delta_{\mathrm{H}} 3.06\right)$ suggested that the 10-acetyl group, $\mathrm{H}-5$, and $\mathrm{H}_{\alpha}-9$ are $\alpha$-oriented, and the 10-methyl group, $\mathrm{C}-5$ prop-1-en-2-yl group, and $\mathrm{H}_{\beta}-9$ are $\beta$-oriented. According to the above data, the structure of 2 was elucidated as 1-oxocurzerenone. This was further confirmed by the ${ }^{1} \mathrm{H}-{ }^{1} \mathrm{H}-\mathrm{COSY}$, NOESY (Figure 4), DEPT, HSQC, and HMBC (Figure 4) techniques.

Table $1 .{ }^{1} \mathrm{H}$ - and ${ }^{13} \mathrm{C}-\mathrm{NMR}$ data of $\mathbf{1 - 3}$.

\begin{tabular}{|c|c|c|c|c|c|c|}
\hline \multirow{2}{*}{ Position } & \multicolumn{2}{|l|}{$1^{a}$} & \multicolumn{2}{|c|}{$2^{a}$} & \multicolumn{2}{|l|}{$3^{b}$} \\
\hline & $\delta_{H}$ & $\delta_{C}$ & $\delta_{H}$ & $\delta_{C}$ & $\delta_{H}$ & $\delta_{C}$ \\
\hline 1 & $5.82 \mathrm{dd}(17.5,11.0)$ & 145.5 & & 212.1 & $5.78 \mathrm{dd}(17.6,10.6)$ & 145.5 \\
\hline 2 & $\begin{array}{l}4.96 \mathrm{~d}(17.5) \\
4.97 \mathrm{~d}(11.0)\end{array}$ & 115.6 & $2.13 \mathrm{~s}$ & 25.6 & $4.74-4.99 \mathrm{~m}$ & 115.7 \\
\hline 3 & $\begin{array}{l}4.76 \mathrm{~s} \\
5.01 \mathrm{~s}\end{array}$ & 113.0 & $\begin{array}{l}4.75 \mathrm{~s} \\
5.01 \mathrm{~s}\end{array}$ & 113.0 & $4.74-4.99 \mathrm{~m}$ & 113.0 \\
\hline 4 & & 141.1 & & 141.0 & & 141.1 \\
\hline 5 & $3.02 \mathrm{~s}$ & 64.1 & $3.22 \mathrm{~s}$ & 61.9 & $3.00 \mathrm{~s}$ & 64.1 \\
\hline 6 & & 194.0 & & 194.0 & & 194.0 \\
\hline 7 & & 120.2 & & 120.1 & & 120.2 \\
\hline 8 & & 165.6 & & 165.6 & & 165.6 \\
\hline $9 \alpha$ & $2.79 \mathrm{~d}(17.5)$ & 33.6 & $2.94 \mathrm{~d}(17.5)$ & 28.5 & $2.83 \mathrm{~d}(17.6)$ & 33.6 \\
\hline $9 \beta$ & $2.91 \mathrm{~d}(17.5)$ & & $3.06 \mathrm{~d}(17.5)$ & & $2.83 \mathrm{~d}(17.6)$ & \\
\hline 10 & & 42.9 & & 46.2 & & 42.9 \\
\hline 11 & & 116.0 & & 119.3 & & 119.3 \\
\hline 12 & $7.09 \mathrm{~s}$ & 139.6 & $7.08 \mathrm{~s}$ & 139.6 & 7.08 br s & 139.6 \\
\hline 13 & $4.86 \mathrm{~s}$ & 52.2 & $2.18 \mathrm{~d}(1.5)$ & 9.0 & 2.16 br s & 9.1 \\
\hline 14 & $1.19 \mathrm{~s}$ & 25.0 & $1.18 \mathrm{~s}$ & 16.2 & $1.17 \mathrm{~s}$ & 25.0 \\
\hline 15 & $1.84 \mathrm{~s}$ & 24.9 & $1.83 \mathrm{~s}$ & 24.7 & $1.83 \mathrm{br} \mathrm{s}$ & 24.9 \\
\hline
\end{tabular}

${ }^{\text {a }}$ Recorded in $\mathrm{CDCl}_{3}$ at $500 \mathrm{MHz}\left({ }^{1} \mathrm{H}\right)$ and $125 \mathrm{MHz}\left({ }^{13} \mathrm{C}\right) \cdot{ }^{\mathrm{b}}$ Recorded in $\mathrm{CDCl}_{3}$ at $300 \mathrm{MHz}\left({ }^{1} \mathrm{H}\right)$ and $75 \mathrm{MHz}$





(a)

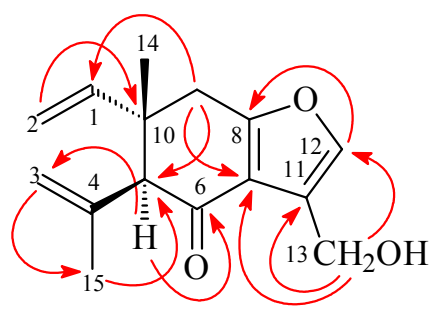

(b)

Figure 3. Key (a) NOESY and (b) HMBC correlations of $\mathbf{1}$.

The known isolates were readily identified by a comparison of physical and spectroscopic data $\left(\mathrm{UV}, \mathrm{IR},{ }^{1} \mathrm{H}-\mathrm{NMR},[\alpha]_{\mathrm{D}}\right.$, and MS) with corresponding authentic samples or literature values, and this included four sesquiterpenoids, curzerenone (3) [5,6], germacrone (4) [7], curcolone (5) [8], and procurcumenol (6) [9], a flavonoid, ermanin (7) [10], a curcuminoid, curcumin (8) [11], a triacylglycerol, 1-oleoyl-2,3-distearoylglycerol (9) [12,13], and six steroids, a mixture of $\beta$-sitosterol (10) and stigmasterol (11) [14,15], a mixture of stigmast-4-en-3,6-dione (12) and stigmasta-4,22-dien-3,6-dione (13) [16], and a mixture of 6 $\beta$-hydroxystigmast-4-en-3-one (14) and 6 $\beta$-hydroxystigmasta-4,22-dien-3-one (15) [17,18]. 


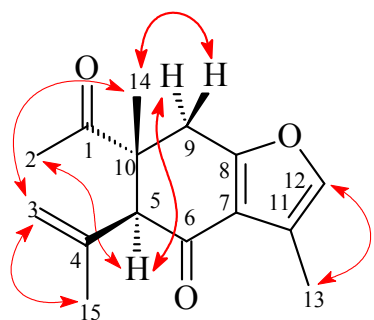

(a)

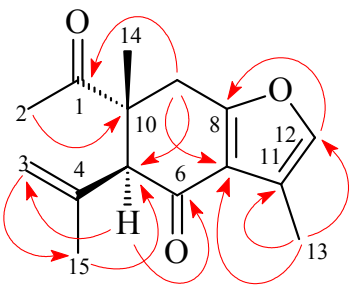

(b)

Figure 4. Key (a) NOESY and (b) HMBC correlations of 2.

The anti-platelet effects of the isolates from the rhizomes of $C$. zedoaria were tested in vitro using the turbidimetric method [19] in washed rabbit platelets induced by collagen $(10 \mu \mathrm{g} / \mathrm{mL})$ and arachidonic acid (AA, $200 \mu \mathrm{M}$ ). The anti-platelet aggregation data are shown in Table 2 . The clinically applied anti-platelet agent, aspirin, was used as the positive control. From the results of our anti-platelet aggregation tests, the following conclusions can be drawn regarding these isolates: (a) 13-Hydroxycurzerenone (1), 1-oxocurzerenone (2), curzerenone (3), germacrone (4), curcolone (5), procurcumenol (6), ermanin (7), curcumin (8), and a mixture of stigmast-4-en-3,6-dione (12) and stigmasta-4,22-dien-3,6-dione (13) exhibited inhibition (with inhibition \% in the range of 21.28-67.58) against collagen-induced platelet aggregation at $100 \mu \mathrm{M}$; (b) compounds 1, 5, 7, 8, and the mixture of 12 and 13 inhibited arachidonic acid (AA)-induced platelet aggregation at $100 \mu \mathrm{M}$ with inhibition \% in the range of 23.44-95.36; (c) ermanin (7) was the most effective among the isolates, with inhibition \% of $67.58 \pm 3.82$ against collagen-induced platelet aggregation at $100 \mu \mathrm{M} ;(\mathrm{d})$ curcumin $(8)$ showed the strongest anti-platelet aggregation activity induced by AA with inhibition $\%$ of $95.36 \pm 1.58$; (e) our study suggests that $C$. zedoaria and its isolates (especially 3,7 , and 8 ) could be further developed as potential candidates for the treatment or prevention of cardiovascular diseases.

Table 2. Inhibitory effects of compounds on the aggregation of washed rabbit platelets induced by collagen and arachidonic acid (AA) ${ }^{\text {a }}$.

\begin{tabular}{|c|c|c|}
\hline \multirow{2}{*}{ Compounds (100 $\mu \mathrm{M})$} & Collagen $(10 \mu \mathrm{g} / \mathrm{mL})$ & Arachidonic Acid $(200 \mu \mathrm{M})$ \\
\hline & \multicolumn{2}{|c|}{ Inhibition (\%) } \\
\hline 13-Hydroxycurzerenone (1) & $22.82 \pm 0.98^{\mathrm{d}}$ & $31.65 \pm 5.33^{c}$ \\
\hline 1-Oxocurzerenone (2) & $23.22 \pm 0.68^{\mathrm{d}}$ & $12.55 \pm 1.59$ \\
\hline Curzerenone (3) & $46.11 \pm 0.38^{\mathrm{e}}$ & $15.41 \pm 1.24^{\mathrm{c}}$ \\
\hline Germacrone (4) & $21.97 \pm 1.15^{\mathrm{d}}$ & $13.22 \pm 2.18$ \\
\hline Curcolone (5) & $35.85 \pm 0.75^{c}$ & $23.44 \pm 3.35^{c}$ \\
\hline Procurcumenol (6) & $21.28 \pm 1.76^{\mathrm{d}}$ & $11.02 \pm 0.86$ \\
\hline Ermanin $(7)$ & $67.58 \pm 3.82^{\mathrm{e}}$ & $34.37 \pm 2.74^{\mathrm{e}}$ \\
\hline Curcumin (8) & $31.57 \pm 2.10^{\mathrm{e}}$ & $95.36 \pm 1.58^{\mathrm{e}}$ \\
\hline 1-Oleoyl-2,3-distearoylglycerol (9) & $4.10 \pm 0.77$ & $3.97 \pm 0.27$ \\
\hline Mixture of $\beta$-sitosterol (10) and stigmasterol (11) & $3.08 \pm 0.43$ & $4.13 \pm 0.64$ \\
\hline $\begin{array}{l}\text { Mixture of stigmast-4-en-3,6-dione (12) and } \\
\text { stigmasta-4,22-dien-3,6-dione (13) }\end{array}$ & $42.71 \pm 2.11^{\mathrm{c}}$ & $23.92 \pm 2.31^{\mathrm{d}}$ \\
\hline $\begin{array}{l}\text { Mixture of } 6 \beta \text {-hydroxystigmast- } 4 \text {-en-3-one (14) } \\
\text { and } 6 \beta \text {-hydroxystigmasta- } 4,22 \text {-dien-3-one (15) }\end{array}$ & $8.21 \pm 1.06$ & $7.53 \pm 0.95$ \\
\hline Aspirin $\mathrm{b}$ & $5.20 \pm 0.35^{\mathrm{e}}$ & $100.0 \pm 0.0^{\mathrm{e}}$ \\
\hline
\end{tabular}

a Platelets were preincubated with each compound or dimethyl sulfoxide (DMSO) $\left(0.5 \%\right.$, control) at $37^{\circ} \mathrm{C}$ for $3 \mathrm{~min}$, then the inducer collagen or arachidonic acid (AA) was added. Results are presented as averages \pm SEM $(n=3-4) .{ }^{\mathrm{b}}$ Aspirin was used as the positive control. ${ }^{\mathrm{c}} p<0.05{ }^{\mathrm{d}} p<0.01$; ${ }^{\mathrm{e}} p<0.001$ compared to control. 


\section{Materials and Methods}

\subsection{General Experimental Procedures}

Melting points were determined on a Yanaco micro-melting point apparatus and were uncorrected. Optical rotations were measured using a Jasco DIP-370 polarimeter (Japan Spectroscopic Corporation, Tokyo, Japan) in $\mathrm{CHCl}_{3}$. Ultraviolet (UV) spectra were obtained on a Jasco UV-240 spectrophotometer. Infrared (IR) spectra (neat or KBr) were recorded on a Perkin Elmer 2000 FT-IR spectrometer (Perkin Elmer Corporation, Norwalk, CT, USA). Nuclear magnetic resonance (NMR) spectra, including correlation spectroscopy (COSY), nuclear Overhauser effect spectrometry (NOESY), heteronuclear multiple-bond correlation (HMBC), and heteronuclear single-quantum coherence (HSQC) experiments were acquired using a Varian Unity 400 or a Varian Inova 500 spectrometer (Varian Inc., Palo Alto, CA, USA) operating at 400 and $500 \mathrm{MHz}\left({ }^{1} \mathrm{H}\right)$ and 100 and $125 \mathrm{MHz}\left({ }^{13} \mathrm{C}\right)$, respectively, with chemical shifts given in ppm $(\delta)$ using tetramethylsilane (TMS) as an internal standard. Electrospray ionization (ESI) and high-resolution electrospray ionization (HR-ESI)-mass spectra were recorded on a Bruker APEX II (Bruker, Bremen, Germany) or a VG Platform Electrospray ESI/MS mass spectrometer (Fison, Villeurbanne, France). Silica gel (70-230, 230-400 mesh, Merck) was used for column chromatography (CC). Silica gel 60 F-254 (Merck, Darmstadt, Germany) was used for thin-layer chromatography (TLC) and preparative thin-layer chromatography (PTLC).

\subsection{Plant Material}

The rhizomes of C. zedoaria were collected from Yanpu Township, Pingtung County, Taiwan, in September 2012 and identified by Prof. Jih-Jung Chen. A voucher specimen (CZ-201209) was deposited in the Department of Pharmacy, Tajen University, Pingtung, Taiwan.

\subsection{Extraction and Isolation}

The dried rhizomes $(3.2 \mathrm{~kg}$ ) of C. zedoaria were extracted three times with $\mathrm{MeOH}$ (12 L each) for 3 days. The extract was concentrated under reduced pressure at $35^{\circ} \mathrm{C}$, and the residue $(285 \mathrm{~g})$ was partitioned between EtOAc and $\mathrm{H}_{2} \mathrm{O}(1: 1)$ to provide the EtOAc-soluble fraction (fraction A; $98 \mathrm{~g}$ ). The $\mathrm{H}_{2} \mathrm{O}$-soluble fraction was further extracted with $\mathrm{BuOH}$, and the $\mathrm{BuOH}$-soluble part (fraction $\mathrm{B}$; $89 \mathrm{~g}$ ) and the $\mathrm{H}_{2} \mathrm{O}$-soluble one (fraction $\mathrm{C} ; 95 \mathrm{~g}$ ) were separated. Fraction A (98 g) was purified by $\mathrm{CC}\left(4.4 \mathrm{~kg}\right.$ of $\mathrm{SiO}_{2}, 70-230$ mesh; $n$-hexane/acetone gradient) to afford 12 fractions: A1-A12. Fraction A3 (7.8 g) was subjected to CC (355 g of $\mathrm{SiO}_{2}, 230-400$ mesh; $n$-hexane/acetone 30:1-0:1, $800 \mathrm{~mL}-$ fractions) to give 10 subfractions: A3-1-A3-10. Fraction A3-4 (155 mg) was purified by preparative TLC $\left(\mathrm{SiO}_{2} ; \mathrm{CHCl}_{3}\right.$ /acetone 50:1) to obtain 1-oleoyl-2,3-distearoylglycerol (9) (4.2 mg) $\left(R_{\mathrm{f}}=0.72\right)$. Fraction A3-5 $(176 \mathrm{mg})$ was further purified by preparative TLC $\left(\mathrm{SiO}_{2} ; n\right.$-hexane/EtOAc $35: 1)$ to obtain germacrone $(4)(5.2 \mathrm{mg})\left(R_{\mathrm{f}}=0.48\right)$. Fraction A3-6 (185 $\left.\mathrm{mg}\right)$ was further purified by preparative TLC $\left(\mathrm{SiO}_{2} ; \mathrm{CHCl}_{3} /\right.$ acetone 60:1) to afford curzerenone (3) (4.6 mg) $\left(R_{\mathrm{f}}=0.69\right)$. Fraction A4 (8.1 g) was subjected to $\mathrm{CC}\left(365 \mathrm{~g}\right.$ of $\mathrm{SiO}_{2}, 230-400$ mesh; $n$-hexane/EtOAc 20:1-0:1, 900 mL-fractions) to give 11 subfractions: A4-1-A4-11. Part (154 mg) of fraction A4-2 was further purified by preparative TLC $\left(\mathrm{SiO}_{2} ; n\right.$-hexane/EtOAc 7:1) to obtain 1-oxocurzerenone (2) (4.0 mg). Part (164 mg) of fraction A4-3 was further purified by preparative TLC $\left(\mathrm{SiO}_{2} ; n\right.$-hexane/acetone 5:1) to obtain curcolone (5) $(4.5 \mathrm{mg})\left(R_{\mathrm{f}}=0.33\right)$. Part $(171 \mathrm{mg})$ of fraction A4-5 was further purified by preparative $\mathrm{TLC}\left(\mathrm{SiO}_{2} ;\right.$ n-hexane/EtOAc, 8:1) to yield procurcumenol (6) (5.4 mg). Fraction A5 (7.5 g) was subjected to CC (340 $\mathrm{g}$ of $\mathrm{SiO}_{2}, 230-400$ mesh; $\mathrm{CHCl}_{3}$ /acetone 15:1-0:1, $950 \mathrm{~mL}$-fractions) to afford nine subfractions: A5-1-A5-9. Fraction A5-4 (365 mg) was purified by MPLC (silica column, $\mathrm{CHCl}_{3} /$ acetone, 10:1-0:1) to afford six subfractions (each $160 \mathrm{~mL}$, A5-4-1-A5-4-6). Fraction A5-4-3 (52 mg) was purified by preparative TLC (silica gel, $\mathrm{CH}_{2} \mathrm{Cl}_{2}$ /acetone, 30:1) to obtain 13-hydroxycurzerenone (1) (3.3 $\mathrm{mg}$ ). Fraction A5-4-4 (58 mg) was further purified by preparative TLC $\left(\mathrm{SiO}_{2} ; \mathrm{CHCl}_{3} /\right.$ acetone, 50:1) to afford a mixture of $\beta$-sitosterol (10) and stigmasterol (11) (14.3 mg) $\left(R_{\mathrm{f}}=0.56\right)$. Part (146 mg) of fraction A5-6 was further purified by preparative TLC $\left(\mathrm{SiO}_{2} ; n\right.$-hexane/acetone, 10:1) to obtain a 
mixture of stigmast-4-en-3,6-dione (12) and stigmasta-4,22-dien-3,6-dione (13) $(11.8 \mathrm{mg})\left(R_{\mathrm{f}}=0.38\right)$. Fraction A7 (8.1 g) was subjected to CC (420 g of $\mathrm{SiO}_{2}, 230-400$ mesh; $\mathrm{CH}_{2} \mathrm{Cl}_{2}$ /acetone 10:1-0:1, 950 mL-fractions) to afford 10 subfractions: A7-1-A7-10. Part (154 mg) of fraction A7-6 was purified by preparative TLC $\left(\mathrm{SiO}_{2} ; n\right.$-hexane/acetone, $\left.8: 1\right)$ to obtain a mixture of $6 \beta$-hydroxystigmast-4-en-3-one (14) and 6 $\beta$-hydroxystigmasta-4,22-dien-3-one (15) $(10.5 \mathrm{mg})\left(R_{\mathrm{f}}=0.22\right)$. Fraction A7-7 (338 mg) was purified by MPLC (silica column, $\mathrm{CHCl}_{3} / \mathrm{MeOH}, 10: 1-0: 1$ ) to afford eight subfractions (each $110 \mathrm{~mL}$, A7-7-1-A7-7-8). Fraction A7-7-4 (48 mg) was purified by preparative TLC (silica gel, $\mathrm{CHCl}_{3} /$ acetone, $1: 1)$ to yield ermanin $\left(=3,4^{\prime}\right.$-dimethoxykaempferol) $(7)(4.5 \mathrm{mg})\left(R_{\mathrm{f}}=0.78\right)$. Fraction A8 $(6.9 \mathrm{~g})$ was subjected to CC (315 g of $\mathrm{SiO}_{2}, 230-400$ mesh; $\mathrm{CH}_{2} \mathrm{Cl}_{2} / \mathrm{MeOH}$ 8:1-0:1, $800 \mathrm{~mL}$ fractions) to afford 11 subfractions: A8-1-A8-11. Part (127 mg) of fraction A8-2 was further purified by preparative TLC $\left(\mathrm{SiO}_{2} ; \mathrm{CHCl}_{3}\right.$ /acetone, $\left.15: 1\right)$ to yield curcumin $(8)(4.7 \mathrm{mg})\left(R_{\mathrm{f}}=0.68\right)$. Part (136 mg) of fraction A8-3 was further purified by preparative TLC $\left(\mathrm{SiO}_{2} ; \mathrm{CH}_{2} \mathrm{Cl}_{2}\right.$ /acetone, 1:1) to afford ermanin (7) (3.5 $\left.\mathrm{mg}\right)$ $\left(R_{\mathrm{f}}=0.79\right)$.

\subsubsection{3-Hydroxycurzerenone (1)}

Yellowish oil. $[\alpha]_{\mathrm{D}}^{25}=+1.5^{\circ}\left(c 0.16, \mathrm{CHCl}_{3}\right)$. UV $(\mathrm{MeOH}): \lambda_{\max }(\log \varepsilon)=272$ (3.32) nm. IR (neat): $v_{\max }=3450(\mathrm{OH}), 1678(\mathrm{C}=\mathrm{O}) \mathrm{cm}^{-1} .{ }^{1} \mathrm{H}-\mathrm{NMR}$ and ${ }^{13} \mathrm{C}-\mathrm{NMR}$ data $\left(500 / 125 \mathrm{MHz}\right.$, in $\left.\mathrm{CDCl}_{3}\right)$ were shown in Table 1. ESI-MS: $m / z=269[\mathrm{M}+\mathrm{Na}]^{+}$. HR-ESI-MS: $m / z=269.1157[\mathrm{M}+\mathrm{Na}]^{+}$(calcd. for $\mathrm{C}_{15} \mathrm{H}_{18} \mathrm{O}_{3} \mathrm{Na}$ : 269.1154) (Supplementary Materials).

\subsubsection{1-Oxocurzerenone (2)}

Yellowish oil. $[\alpha]_{\mathrm{D}}^{25}=+2.1^{\circ}\left(c 0.14, \mathrm{CHCl}_{3}\right)$. UV $(\mathrm{MeOH}): \lambda_{\max }(\log \varepsilon)=273(3.41) \mathrm{nm}$. IR (neat): $v_{\max }=1706(\mathrm{C}=\mathrm{O}), 1675(\mathrm{C}=\mathrm{O}) \mathrm{cm}^{-1} \cdot{ }^{1} \mathrm{H}-\mathrm{NMR}$ and ${ }^{13} \mathrm{C}-\mathrm{NMR}$ data $\left(500 / 125 \mathrm{MHz}\right.$, in $\left.\mathrm{CDCl}_{3}\right)$ were shown in Table 1. ESI-MS: $m / z=269[\mathrm{M}+\mathrm{Na}]^{+}$. HR-ESI-MS: $m / z=269.1153[\mathrm{M}+\mathrm{Na}]^{+}$(calcd. for $\mathrm{C}_{15} \mathrm{H}_{18} \mathrm{O}_{3} \mathrm{Na}$ : 269.1154) (Supplementary Materials).

\subsubsection{Curzerenone (3)}

Yellowish oil. UV $(\mathrm{MeOH}): \lambda_{\max }(\log \varepsilon)=272(3.30) \mathrm{nm}$. IR (neat): $v_{\max }=1676(\mathrm{C}=\mathrm{O}) \mathrm{cm}^{-1}$. ${ }^{1} \mathrm{H}-\mathrm{NMR}\left(\mathrm{CDCl}_{3}, 500 \mathrm{MHz}\right): \delta=1.19(3 \mathrm{H}, \mathrm{s}, \mathrm{H}-14), 1.84(3 \mathrm{H}, \mathrm{s}, \mathrm{H}-15), 2.19(3 \mathrm{H}, \mathrm{d}, J=1.5 \mathrm{~Hz}, \mathrm{H}-13)$, $2.79(1 \mathrm{H}, \mathrm{d}, J=17.5 \mathrm{~Hz}, \mathrm{H}-9 \alpha), 2.91(1 \mathrm{H}, \mathrm{d}, J=17.5 \mathrm{~Hz}, \mathrm{H}-9 \beta), 3.01(1 \mathrm{H}, \mathrm{s}, \mathrm{H}-5), 4.76(1 \mathrm{H}, \mathrm{s}, \mathrm{H}-3), 4.96$ $(1 \mathrm{H}, \mathrm{d}, J=17.5 \mathrm{~Hz}, \mathrm{H}-2), 4.97(1 \mathrm{H}, \mathrm{d}, J=11.0 \mathrm{~Hz}, \mathrm{H}-2), 5.01(1 \mathrm{H}, \mathrm{s}, \mathrm{H}-3), 5.81(1 \mathrm{H}, \mathrm{dd}, J=17.5,11.0 \mathrm{~Hz}$, H-1), 7.09 (1H, s, H-12). ESI-MS: $m / z=231[\mathrm{M}+\mathrm{H}]^{+}$.

\subsubsection{Germacrone (4)}

Colorless crystals ( $n$-hexane), mp. 53-55 ${ }^{\circ} \mathrm{C}$. UV $(\mathrm{MeOH}): \lambda_{\max }(\log \varepsilon)=272(3.30) \mathrm{nm}$. IR $(\mathrm{KBr})$ : $v_{\max }=1676(\mathrm{C}=\mathrm{O}) \mathrm{cm}^{-1} .{ }^{1} \mathrm{H}-\mathrm{NMR}\left(\mathrm{CDCl}_{3}, 500 \mathrm{MHz}\right): \delta=1.19(3 \mathrm{H}, \mathrm{s}, \mathrm{H}-14), 1.84(3 \mathrm{H}, \mathrm{s}, \mathrm{H}-15), 2.19$ $(3 \mathrm{H}, \mathrm{d}, J=1.5 \mathrm{~Hz}, \mathrm{H}-13), 2.79(1 \mathrm{H}, \mathrm{d}, J=17.5 \mathrm{~Hz}, \mathrm{H}-9 \alpha), 2.91(1 \mathrm{H}, \mathrm{d}, J=17.5 \mathrm{~Hz}, \mathrm{H}-9 \beta), 3.01(1 \mathrm{H}, \mathrm{s}$, H-5), $4.76(1 \mathrm{H}, \mathrm{s}, \mathrm{H}-3), 4.96(1 \mathrm{H}, \mathrm{d}, J=17.5 \mathrm{~Hz}, \mathrm{H}-2), 4.97(1 \mathrm{H}, \mathrm{d}, J=11.0 \mathrm{~Hz}, \mathrm{H}-2), 5.01(1 \mathrm{H}, \mathrm{s}, \mathrm{H}-3)$, $5.81(1 \mathrm{H}, \mathrm{dd}, J=17.5,11.0 \mathrm{~Hz}, \mathrm{H}-1), 7.09(1 \mathrm{H}, \mathrm{s}, \mathrm{H}-12)$. ESI-MS: $m / z=231[\mathrm{M}+\mathrm{H}]^{+}$.

\subsubsection{Curcolone (5)}

Colorless crystals ( $n$-hexane-EtOAc), mp. $151-153^{\circ} \mathrm{C} .[\alpha]_{\mathrm{D}}^{25}=+13.9^{\circ}\left(c 0.13, \mathrm{CHCl}_{3}\right)$. UV $(\mathrm{MeOH})$ : $\lambda_{\max }(\log \varepsilon)=214$ (3.85), 259 (3.38), 286 (3.37) nm. IR (KBr): $v_{\max }=3445(\mathrm{OH}), 1651(\mathrm{C}=\mathrm{O}) \mathrm{cm}^{-1}$. ${ }^{1} \mathrm{H}-\mathrm{NMR}\left(\mathrm{CDCl}_{3}, 500 \mathrm{MHz}\right): \delta=1.13(3 \mathrm{H}, \mathrm{s}, \mathrm{H}-14), 1.70-1.81(2 \mathrm{H}, \mathrm{m}, \mathrm{H}-2), 2.05$ (3H, s, H-15), $2.24(3 \mathrm{H}$, $\mathrm{d}, J=1.5 \mathrm{~Hz}, \mathrm{H}-13), 2.30-2.35(2 \mathrm{H}, \mathrm{m}, \mathrm{H}-3), 2.77(1 \mathrm{H}, \mathrm{d}, J=17.0 \mathrm{~Hz}, \mathrm{H}-9), 3.11(1 \mathrm{H}, \mathrm{d}, J=17.0 \mathrm{~Hz}, \mathrm{H}-9)$, $3.82(1 \mathrm{H}, \mathrm{dd}, J=10.8,3.8 \mathrm{~Hz}, \mathrm{H}-1), 7.07(1 \mathrm{H}, \mathrm{s}, \mathrm{H}-12)$. ESI-MS: $m / z=269[\mathrm{M}+\mathrm{Na}]^{+}$. 


\subsubsection{Procurcumenol (6)}

Viscous oil. $[\alpha]_{\mathrm{D}}^{24}=+218.5^{\circ}\left(c 0.15, \mathrm{CHCl}_{3}\right)$. UV (MeOH): $\lambda_{\max }(\log \varepsilon)=248(3.90), 275$ (3.75) nm. IR (neat): $v_{\max }=3430(\mathrm{OH}), 1646(\mathrm{C}=\mathrm{O}) \mathrm{cm}^{-1} .{ }^{1} \mathrm{H}-\mathrm{NMR}\left(\mathrm{CDCl}_{3}, 500 \mathrm{MHz}\right): \delta=1.24(3 \mathrm{H}, \mathrm{s}, \mathrm{H}-14), 1.75$ $(3 \mathrm{H}, \mathrm{s}, \mathrm{H}-13), 1.78$ (3H, s, H-12), 1.88 (3H, s, H-15), 2.18 (1H, dd, J = 16.0, $13.0 \mathrm{~Hz}, \mathrm{H}-6 \alpha), 2.38(1 \mathrm{H}, \mathrm{ddd}$, $J=10.5,10.0,10.0 \mathrm{~Hz}, \mathrm{H}-1), 2.61(1 \mathrm{H}, \mathrm{br} d, J=16.0 \mathrm{~Hz}, \mathrm{H}-6 \beta), 5.88(1 \mathrm{H}$, br s, H-9). ESI-MS: $m / z=235$ $[\mathrm{M}+\mathrm{H}]^{+}$.

\subsubsection{Ermanin (=3,4'-Dimethoxykaempferol) $(7)$}

Yellowish needles (EtOAc), mp. $233-235{ }^{\circ} \mathrm{C}$. UV (MeOH): $\lambda_{\max }=266,296$ (sh), $345 \mathrm{~nm}$. IR (KBr): $v_{\max }=3990(\mathrm{OH}), 1657(\mathrm{C}=\mathrm{O}) \mathrm{cm}^{-1} .{ }^{1} \mathrm{H}-\mathrm{NMR}\left(\mathrm{CDCl}_{3}, 500 \mathrm{MHz}\right): \delta=3.86(3 \mathrm{H}, \mathrm{s}, \mathrm{OMe}-3), 3.90(3 \mathrm{H}$, s, OMe-4' $\left.{ }^{\prime}\right), 6.28(1 \mathrm{H}, \mathrm{d}, J=2.0 \mathrm{~Hz}, \mathrm{H}-6), 6.41(1 \mathrm{H}, \mathrm{d}, J=2.0 \mathrm{~Hz}, \mathrm{H}-8), 7.03\left(2 \mathrm{H}, \mathrm{d}, J=9.0 \mathrm{~Hz}, \mathrm{H}-3^{\prime}\right.$ and $\left.\mathrm{H}-5^{\prime}\right), 8.07\left(2 \mathrm{H}, \mathrm{d}, J=9.0 \mathrm{~Hz}, \mathrm{H}-2^{\prime}\right.$ and $\left.\mathrm{H}-6^{\prime}\right), 12.73\left(1 \mathrm{H}, \mathrm{s}, \mathrm{D}_{2} \mathrm{O}\right.$ exchangeable, OH-5). ESI-MS: $m / z=315[\mathrm{M}+\mathrm{H}]^{+}$.

\subsubsection{Curcumin (8)}

Orange crystals $\left(\mathrm{CH}_{2} \mathrm{Cl}_{2}-\mathrm{MeOH}\right), \mathrm{mp} .182-183{ }^{\circ} \mathrm{C}$. UV $(\mathrm{MeOH}): \lambda_{\max }(\log \varepsilon)=422(4.51) \mathrm{nm}$. IR $(\mathrm{KBr}): v_{\max }=3417(\mathrm{OH}), 1629(\mathrm{C}=\mathrm{O}) \mathrm{cm}^{-1} .{ }^{1} \mathrm{H}-\mathrm{NMR}\left(\mathrm{CDCl}_{3}, 400 \mathrm{MHz}\right): \delta=3.96\left(6 \mathrm{H}, \mathrm{s}, \mathrm{OMe}-3^{\prime}\right.$ and OMe-3' $\left.3^{\prime \prime}\right), 5.81\left(1 \mathrm{H}, \mathrm{s}, \mathrm{H}-4\right.$, this proton signal assignable to an enol form), $5.86\left(2 \mathrm{H}, \mathrm{br} \mathrm{s}, \mathrm{OH}-4^{\prime}\right.$ and $\left.\mathrm{OH}-4^{\prime \prime}\right), 6.48\left(2 \mathrm{H}, \mathrm{d}, J=15.6 \mathrm{~Hz}, \mathrm{H}-2\right.$ and H-6) $6.94\left(2 \mathrm{H}, \mathrm{d}, J=8.0 \mathrm{~Hz}, \mathrm{H}-5^{\prime}\right.$ and $\left.\mathrm{H}-5^{\prime \prime}\right), 7.06(2 \mathrm{H}, \mathrm{d}$, $J=2.0 \mathrm{~Hz}, \mathrm{H}-2^{\prime}$ and $\left.\mathrm{H}-2^{\prime \prime}\right), 7.13\left(2 \mathrm{H}, \mathrm{dd}, J=8.0,2.0 \mathrm{~Hz}, \mathrm{H}-6^{\prime}\right.$ and $\left.\mathrm{H}-6^{\prime \prime}\right), 7.60(2 \mathrm{H}, \mathrm{d}, J=15.6 \mathrm{~Hz}, \mathrm{H}-1$ and H-7). ESI-MS: $m / z=369[\mathrm{M}+\mathrm{H}]^{+}$.

\subsubsection{1-Oleoyl-2,3-distearoylglycerol (9)}

Colorless oil. $[\alpha]_{\mathrm{D}}^{25}=+3.6^{\circ}\left(c 0.2, \mathrm{CHCl}_{3}\right)$. IR (neat): $v_{\max }=1744(\mathrm{C}=\mathrm{O}) \mathrm{cm}^{-1} .{ }^{1} \mathrm{H}-\mathrm{NMR}\left(\mathrm{CDCl}_{3}\right.$, $500 \mathrm{MHz}): \delta=0.88\left(9 \mathrm{H}, \mathrm{t}, J=7.0 \mathrm{~Hz}, \mathrm{H}-18^{\prime}, \mathrm{H}-18^{\prime \prime}\right.$, and $\left.\mathrm{H}-18^{\prime \prime \prime}\right), 1.26\left(76 \mathrm{H}, \mathrm{br} \mathrm{s}, \mathrm{H}-4^{\prime} \sim \mathrm{H}-7^{\prime}\right.$ and $\mathrm{H}-12^{\prime} \sim \mathrm{H}-17^{\prime}, \mathrm{H}-4^{\prime \prime} \sim \mathrm{H}-17^{\prime \prime}$, and H-4 $\left.{ }^{\prime \prime \prime} \sim \mathrm{H}-17^{\prime \prime \prime}\right), 1.61\left(6 \mathrm{H}, \mathrm{m}, \mathrm{H}-3^{\prime}, \mathrm{H}-3^{\prime \prime}\right.$, and H-3 $\left.{ }^{\prime \prime \prime}\right), 2.01\left(4 \mathrm{H}, \mathrm{m}, \mathrm{H}-8^{\prime}\right.$ and $\left.\mathrm{H}-11^{\prime}\right), 2.31\left(6 \mathrm{H}, \mathrm{t}, J=7.5 \mathrm{~Hz}, \mathrm{H}-2^{\prime}, \mathrm{H}-2^{\prime \prime}\right.$, and H-2 $\left.{ }^{\prime \prime \prime}\right), 4.15(2 \mathrm{H}, \mathrm{dd}, J=12.0,5.5 \mathrm{~Hz}, \mathrm{H}-1 \mathrm{a}$ and H-3a), $4.30(2 \mathrm{H}, \mathrm{dd}, J=12.0,4.0 \mathrm{~Hz}, \mathrm{H}-1 \mathrm{~b}$ and $\mathrm{H}-3 \mathrm{~b}), 5.27(1 \mathrm{H}, \mathrm{m}, \mathrm{H}-2), 5.35\left(2 \mathrm{H}, \mathrm{m}, \mathrm{H}-9^{\prime}\right.$ and $\left.\mathrm{H}-10^{\prime}\right)$. ESI-MS: $m / z=911[\mathrm{M}+\mathrm{Na}]^{+}$.

\subsubsection{Mixture of $\beta$-Sitosterol (10) and Stigmasterol (11) (ratio 4:1)}

Colorless needles $(\mathrm{MeOH}), \mathrm{mp} .133-135^{\circ} \mathrm{C} .[\alpha]_{\mathrm{D}}^{24}=-35.6^{\circ}\left(c 0.24, \mathrm{CHCl}_{3}\right) . \mathrm{IR}(\mathrm{KBr}): v_{\max }=3440$ $(\mathrm{OH}) \mathrm{cm}^{-1} .{ }^{1} \mathrm{H}-\mathrm{NMR}\left(\mathrm{CDCl}_{3}, 500 \mathrm{MHz}\right)$ of $10: \delta=0.68(3 \mathrm{H}, \mathrm{s}, \mathrm{H}-18), 0.81(3 \mathrm{H}, \mathrm{d}, J=6.8 \mathrm{~Hz}, \mathrm{H}-27), 0.83$ $(3 \mathrm{H}, \mathrm{d}, J=6.8 \mathrm{~Hz}, \mathrm{H}-26), 0.85(3 \mathrm{H}, \mathrm{t}, J=7.2 \mathrm{~Hz}, \mathrm{H}-29), 0.92(3 \mathrm{H}, \mathrm{d}, J=6.5 \mathrm{~Hz}, \mathrm{H}-21), 1.01(3 \mathrm{H}, \mathrm{s}, \mathrm{H}-19)$, $3.52(1 \mathrm{H}, \mathrm{m}, \mathrm{H}-3), 5.35(1 \mathrm{H}, \mathrm{d}, J=5.5 \mathrm{~Hz}, \mathrm{H}-6) ;{ }^{1} \mathrm{H}-\mathrm{NMR}\left(\mathrm{CDCl}_{3}, 500 \mathrm{MHz}\right)$ of $11: \delta=0.70(3 \mathrm{H}, \mathrm{s}, \mathrm{H}-18)$, $0.79(3 \mathrm{H}, \mathrm{d}, J=6.8 \mathrm{~Hz}, \mathrm{H}-27), 0.83(3 \mathrm{H}, \mathrm{d}, J=6.8 \mathrm{~Hz}, \mathrm{H}-26), 0.82(3 \mathrm{H}, \mathrm{t}, J=7.2 \mathrm{~Hz}, \mathrm{H}-29), 1.01(3 \mathrm{H}, \mathrm{s}$, $\mathrm{H}-19), 1.02(3 \mathrm{H}, \mathrm{d}, J=6.5 \mathrm{~Hz}, \mathrm{H}-21), 3.52(1 \mathrm{H}, \mathrm{m}, \mathrm{H}-3), 5.01(1 \mathrm{H}, \mathrm{dd}, J=15.5,8.5 \mathrm{~Hz}, \mathrm{H}-23), 5.15(1 \mathrm{H}$, $\mathrm{dd}, J=15.5,8.5 \mathrm{~Hz}, \mathrm{H}-22), 5.35(1 \mathrm{H}, \mathrm{br} \mathrm{d}, J=5.5 \mathrm{~Hz}, \mathrm{H}-6)$. ESI-MS of 10: $m / z=415[\mathrm{M}+\mathrm{H}]^{+}$; ESI-MS of 11: $m / z=413[\mathrm{M}+\mathrm{H}]^{+}$.

\subsubsection{Mixture of Stigmast-4-en-3,6-dione (12) and Stigmasta-4,22-dien-3,6-dione (13) (ratio 4.7:1)}

Light-yellow needles $(\mathrm{MeOH}), \mathrm{mp} .172-174{ }^{\circ} \mathrm{C} .[\alpha]_{\mathrm{D}}^{24}=-38.2^{\circ}\left(c 0.24, \mathrm{CHCl}_{3}\right)$. UV $(\mathrm{MeOH})$ : $\lambda_{\max }(\log \varepsilon)=250(4.16) \mathrm{nm} . \mathrm{IR}(\mathrm{KBr}): v_{\max }=1689(\mathrm{C}=\mathrm{O}) \mathrm{cm}^{-1} \cdot{ }^{1} \mathrm{H}-\mathrm{NMR}\left(\mathrm{CDCl}_{3}, 500 \mathrm{MHz}\right)$ of 12: $\delta=0.72(3 \mathrm{H}, \mathrm{s}, \mathrm{H}-18), 0.81(3 \mathrm{H}, \mathrm{d}, J=7.0 \mathrm{~Hz}, \mathrm{H}-27), 0.83(3 \mathrm{H}, \mathrm{d}, J=7.0 \mathrm{~Hz}, \mathrm{H}-26), 0.86(3 \mathrm{H}, \mathrm{t}, J=7.0 \mathrm{~Hz}$, H-29), $0.94(3 \mathrm{H}, \mathrm{d}, J=6.5 \mathrm{~Hz}, \mathrm{H}-21), 1.17$ (3H, s, H-19), $6.17(1 \mathrm{H}, \mathrm{s}, \mathrm{H}-4) ;{ }^{1} \mathrm{H}-\mathrm{NMR}\left(\mathrm{CDCl}_{3}, 500 \mathrm{MHz}\right)$ of 13: $\delta=0.74(3 \mathrm{H}, \mathrm{s}, \mathrm{H}-18), 0.79(3 \mathrm{H}, \mathrm{d}, J=7.0 \mathrm{~Hz}, \mathrm{H}-27), 0.83(3 \mathrm{H}, \mathrm{d}, J=7.0 \mathrm{~Hz}, \mathrm{H}-26), 0.82(3 \mathrm{H}, \mathrm{t}$, $J=7.0 \mathrm{~Hz}, \mathrm{H}-29), 1.17(3 \mathrm{H}, \mathrm{s}, \mathrm{H}-19), 1.04(3 \mathrm{H}, \mathrm{d}, J=6.5 \mathrm{~Hz}, \mathrm{H}-21), 6.17(1 \mathrm{H}, \mathrm{m}, \mathrm{H}-4), 5.04(1 \mathrm{H}, \mathrm{dd}$, $J=15.5,8.5 \mathrm{~Hz}, \mathrm{H}-23), 5.15(1 \mathrm{H}, \mathrm{dd}, J=15.5,8.5 \mathrm{~Hz}, \mathrm{H}-22)$. ESI-MS of 12: $m / z=449[\mathrm{M}+\mathrm{Na}]^{+}$; ESI-MS of 13: $m / z=447[\mathrm{M}+\mathrm{Na}]^{+}$. 
3.3.12. Mixture of 6 $\beta$-Hydroxystigmast-4-en-3-one (14) and 6 $\beta$-Hydroxystigmasta-4,22-dien-3-one (15) (ratio 3.3:1)

Colorless needles $(\mathrm{MeOH}), \mathrm{mp} . \quad 207-209{ }^{\circ} \mathrm{C} .[\alpha]_{\mathrm{D}}^{24}=+30.2^{\circ}\left(c 0.15, \mathrm{CHCl}_{3}\right)$. UV $(\mathrm{MeOH})$ : $\lambda_{\max }(\log \varepsilon)=246(4.13) \mathrm{nm} . \mathrm{IR}(\mathrm{KBr}): v_{\max }=3430(\mathrm{OH}), 1676(\mathrm{C}=\mathrm{O}) \mathrm{cm}^{-1} .{ }^{1} \mathrm{H}-\mathrm{NMR}\left(\mathrm{CDCl}_{3}\right.$, $500 \mathrm{MHz})$ of 14: $\delta=0.74(3 \mathrm{H}, \mathrm{s}, \mathrm{H}-18), 0.81(3 \mathrm{H}, \mathrm{d}, J=7.0 \mathrm{~Hz}, \mathrm{H}-27), 0.84(3 \mathrm{H}, \mathrm{d}, J=7.0 \mathrm{~Hz}, \mathrm{H}-26), 0.87$ $(3 \mathrm{H}, \mathrm{t}, J=7.0 \mathrm{~Hz}, \mathrm{H}-29), 0.92(3 \mathrm{H}, \mathrm{d}, J=6.5 \mathrm{~Hz}, \mathrm{H}-21), 1.38(3 \mathrm{H}, \mathrm{s}, \mathrm{H}-19), 4.35(1 \mathrm{H}, \mathrm{t}, J=3.0 \mathrm{~Hz}, \mathrm{H}-6)$, $5.82(1 \mathrm{H}, \mathrm{s}, \mathrm{H}-4) ;{ }^{1} \mathrm{H}-\mathrm{NMR}\left(\mathrm{CDCl}_{3}, 500 \mathrm{MHz}\right)$ of 15: $\delta=0.76(3 \mathrm{H}, \mathrm{s}, \mathrm{H}-18), 0.80(3 \mathrm{H}, \mathrm{d}, J=7.0 \mathrm{~Hz}, \mathrm{H}-27)$, $0.81(3 \mathrm{H}, \mathrm{d}, J=7.0 \mathrm{~Hz}, \mathrm{H}-26), 0.85(3 \mathrm{H}, \mathrm{t}, J=7.2 \mathrm{~Hz}, \mathrm{H}-29), 1.38(3 \mathrm{H}, \mathrm{s}, \mathrm{H}-19), 1.02(3 \mathrm{H}, \mathrm{d}, J=6.0 \mathrm{~Hz}$, H-21), $4.35(1 \mathrm{H}, \mathrm{t}, J=3.0 \mathrm{~Hz}, \mathrm{H}-6), 5.03(1 \mathrm{H}, \mathrm{dd}, J=15.0,8.5 \mathrm{~Hz}, \mathrm{H}-23), 5.15(1 \mathrm{H}, \mathrm{dd}, J=15.0,8.5 \mathrm{~Hz}$, H-22), $5.82(1 \mathrm{H}, \mathrm{s}, \mathrm{H}-4)$. ESI-MS of 14: $m / z=429[\mathrm{M}+\mathrm{H}]^{+}$; ESI-MS of 15: $m / z=427[\mathrm{M}+\mathrm{H}]^{+}$.

\subsection{Anti-Platelet Aggregation Test}

Blood was collected from the rabbit marginal vein, anticoagulated with EDTA (6 mM) and centrifuged for $10 \mathrm{~min}$ at $90 \times g$ at room temperature to obtain platelet-rich plasma (PRP). Platelet suspension was prepared from this EDTA-anticoagulated PRP according to the washing procedures described previously [20]. Platelet numbers were counted by a Coulter counter (Model ZM) and adjusted to $4.5 \times 10^{8}$ platelets $/ \mathrm{mL}$. The platelet pellets were finally suspended in Tyrode's solution of the following composition (mM): $\mathrm{NaCl}(136.8), \mathrm{KCl}(2.8), \mathrm{NaHCO}_{3}$ (11.9), $\mathrm{MgCl}_{2}(2.1), \mathrm{NaH}_{2} \mathrm{PO}_{4}$ (0.33), $\mathrm{CaCl}_{2}$ (1.0), and glucose (11.2), containing bovine serum albumin (0.35\%). Platelet aggregation was measured at $37^{\circ} \mathrm{C}$ by the turbidimetric method as described by O'Brien [19] using a Chrono-log Lumi-aggregometer. The platelet suspensions were stirred at $1200 \mathrm{rpm}$. All the tested compounds were dissolved in dimethyl sulfoxide (DMSO). In order to eliminate the effect of the solvent on the aggregation, the final concentration of DMSO was fixed at $0.5 \%$, and did not affect the aggregation measured. Percentages of aggregation were calculated using the absorbance of platelet suspension to represent $0 \%$ aggregation and the absorbance of Tyrode's solution as $100 \%$ aggregation. Aspirin was used as a positive control. Data were analyzed using Student's $t$-test.

Statistical Analysis

Results are expressed as the mean \pm SEM, and comparisons were made using Student's $t$-test. A probability of 0.05 or less was considered significant. The software SigmaPlot (version 11.0, SPSS Inc., Chicago, IL, USA) was used for the statistical analysis.

\section{Conclusions}

Fifteen compounds, including two new sesquiterpenoids, 13-hydroxycurzerenone (1) and 1-oxocurzerenone (2), were isolated from the rhizomes of $C$. zedoaria. The structures of these compounds were established on the basis of spectroscopic data. The anti-platelet effects of the isolates were evaluated by suppressing collagen- and AA-induced platelet aggregation in washed rabbit platelets. The results of anti-platelet aggregation experiments indicate that compounds 1, 3, 5, 7, 8, and the mixture of 12 and 13 can significantly inhibit collagen- and/or AA-induced platelet aggregation at $100 \mu \mathrm{M}$. Ermanin (7) and curcumin (8) were the most effective among the isolated compounds, with inhibition \% of $67.58 \pm 3.82$ and $95.36 \pm 1.58 \mu \mathrm{g} / \mathrm{mL}$, respectively, against collagen- and AA-induced platelet aggregation at $100 \mu \mathrm{M}$. Our study suggests that C. zedoaria and its isolates (especially 3, 7 , and 8) could be further developed as potential candidates for the treatment or prevention of cardiovascular diseases.

Supplementary Materials: Supplementary materials can be found at www.mdpi.com/1420-3049/21/10/1385/s1.

Acknowledgments: This research was supported by grants from the Ministry of Science and Technology, Taiwan (No. NSC 101-2320-B-127-001-MY3 and MOST 105-2320-B-127-002), awarded to J.-J. Chen. This work was also supported by the grants from Zuoying Branch of Kaohsiung Armed Forces General Hospital (ZBH105-25 and ZBH105-26). 
Author Contributions: Jih-Jung Chen designed the research; Jih-Jung Chen, Tung-Han Tsai, Hsiang-Ruei Liao, Li-Chai Chen, Yueh-Hsiung Kuo, Ping-Jyun Sung, Chun-Lin Chen and Chun-Sheng Wei performed the experiments and analyzed the data; and Jih-Jung Chen wrote the paper. All authors have read and approved the final manuscript.

Conflicts of Interest: The authors declare no conflict of interest.

\section{Abbreviations}

$\begin{array}{ll}\text { fMLP } & \text { N-formyl-L-methionyl-L-leucyl-L-phenylalanine } \\ \text { CB } & \text { cytochalasin B } \\ \text { COSY } & \text { correlation spectroscopy } \\ \text { NOESY } & \text { nuclear Overhauser effect spectrometry } \\ \text { HMBC } & \text { heteronuclear multiple-bond correlation } \\ \text { HSQC } & \text { heteronuclear single-quantum coherence } \\ \text { DEPT } & \text { distortionless enhancement by polarization transfer } \\ \text { ESI } & \text { electrospray ionisation } \\ \text { HRESI } & \text { high-resolution electrospray ionization } \\ \text { CC } & \text { column chromatography } \\ \text { TLC } & \text { thin-layer chromatography } \\ \text { MPLC } & \text { medium pressure liquid chromatography }\end{array}$

\section{References}

1. Makabe, H.; Maru, N.; Kuwabara, A.; Kamo, T.; Hirota, M. Anti-inflammatory sesquiterpenes from Curcuma zedoaria. Nat. Prod. Res. 2006, 20, 680-685. [CrossRef] [PubMed]

2. Kasahara, K.; Nomura, S.; Subeki; Matsuura, H.; Yamasaki, M.; Yamato, O.; Maede, Y.; Katakura, K.; Suzuki, M.; Yoshihara, T. Anti-babesial compounds from Curcuma zedoaria. Planta Med. 2005, 71, 482-484. [CrossRef] [PubMed]

3. Syu, W.J.; Shen, C.C.; Don, M.J.; Ou, J.C.; Lee, G.H.; Sun, C.M. Cytotoxicity of curcuminoids and some novel compounds from Curcuma zedoaria. J. Nat. Prod. 1998, 61, 1531-1534. [CrossRef] [PubMed]

4. Chen, Z.; Wei, Y.; Li, X.; Peng, C.; Long, Z. Antifungal activity and mechanism of major compound isolated from hexane extract of Curcuma zedoaria. Asian J. Chem. 2013, 25, 6597-6600.

5. Fukushima, S.; Kuroyanagi, M.; Ueno, A.; Akahori, Y.; Saiki, Y. Structure of curzerenone, a new sesquiterpene from Curcuma zedoaria. Yakugaku Zasshi 1970, 90, 863-869. [PubMed]

6. Dekebo, A.; Dagne, E.; Sterner, O. Furanosesquiterpenes from Commiphora sphaerocarpa and related adulterants of true myrrh. Fitoterapia 2002, 73, 48-55. [CrossRef]

7. Firman, K.; Kinoshita, T.; Itai, A.; Sankawa, U. Terpenoids from Curcuma heyneana. Phytochemistry 1988, 27, 3887-3892. [CrossRef]

8. Hikino, H.; Sakurai, Y.; Takemoto, T. Structure of curcolone. Chem. Pharm. Bull. 1967, 15, 1065-1066. [CrossRef] [PubMed]

9. Ohshiro, M.; Kuroyanagi, M.; Ueno, A. Structures of sesquiterpenes from Curcuma longa. Phytochemistry 1990, 29, 2201-2205. [CrossRef]

10. Nakatani, N.; Jitoe, A.; Masuda, T.; Yonemori, S. Flavonoid constituents of Zingiber zerumbet Smith. Agric. Biol. Chem. 1991, 55, 455-460. [CrossRef]

11. Sun, R.; Sacalis, J.N.; Chin, C.K.; Still, C.C. Bioactive aromatic compounds from leaves and stems of Vanilla fragrans. J. Agr. Food Chem. 2001, 49, 5161-5164. [CrossRef]

12. Jie, M.S.F.L.K.; Lam, C.C. ${ }^{13}$ C-NMR studies of polyunsaturated triacylglycerols of type AAA and mixed triacylglycerols containing saturated, acetylenic and ethylenic acyl groups. Chem. Phys. Lipids 1995, 78. [CrossRef]

13. Kristinsson, B.; Linderborg, K.M.; Kallio, H.; Haraldsson, G.G. Synthesis of enantiopure structured triacylglycerols. Tetrahedron: Asymmetry 2014, 25, 125-132. [CrossRef]

14. Tao, R.; Wang, C.Z.; Kong, Z.W. Antibacterial/antifungal activity and synergistic interactions between polyprenols and other lipids isolated from Ginkgo Biloba L. leaves. Molecules 2013, 18, 2166-2182. [CrossRef] [PubMed] 
15. Ahmed, E.; Sharif, A.; Hussain, S.; Malik, A.; Mukhtar-Ul-Hassan; Munawar, M.A.; Nagra, S.A.; Anwar, J.; Ashraf, M.; Afza, N.; Athar, M. Phytochemical and antimicrobial studies of Grewia tenax. J. Chem. Soc. Pak. 2011, 33, 676-681.

16. Cui, J.G.; Fan, L.; Huang, L.L.; Liu, H.L.; Zhou, A.M. Synthesis and evaluation of some steroidal oximes as cytotoxic agents: Structure/activity studies (I). Steroids 2009, 74, 62-72. [CrossRef] [PubMed]

17. Sun, X.B.; Zhao, P.H.; Xu, Y.J.; Sun, L.M.; Cao, M.A.; Yuan, C.S. Chemical constituents from the roots of Polygonum bistorta. Chem. Nat. Compd. 2007, 43, 563-566. [CrossRef]

18. Ayyad, S.N. A new cytotoxic stigmastane steroid from Pistia stratiotes. Pharmazie 2002, 57, 212-214. [PubMed]

19. O'Brien, J.R. Platelet aggregation II.Some results from a new method of study. J. Clin. Path. 1962, 15, 452-455.

20. Teng, C.M.; Chen, W.Y.; Ko, W.C.; Ouyang, C. Antiplatelet effect of butylidenephthalide. Biochem. Biophys. Acta 1987, 924, 375-382. [CrossRef]

Sample Availability: Samples of the compounds are available from the authors.

(C) 2016 by the authors; licensee MDPI, Basel, Switzerland. This article is an open access article distributed under the terms and conditions of the Creative Commons Attribution (CC-BY) license (http://creativecommons.org/licenses/by/4.0/). 